\title{
Neutral modes of a two-dimensional vortex and their link to persistent cat's eyes
}

\author{
M. R. Turner, ${ }^{1, a)}$ Andrew D. Gilbert, ${ }^{1}$ and Andrew P. Bassom ${ }^{2}$ \\ ${ }^{1}$ Mathematics Research Institute, School of Engineering, Computing and Mathematics, University of Exeter, \\ Exeter EX4 4QF, United Kingdom \\ ${ }^{2}$ School of Mathematics and Statistics, University of Western Australia, Crawley 6009, Australia
}

(Received 1 August 2007; accepted 14 December 2007; published online 14 February 2008)

\begin{abstract}
This paper considers the relaxation of a smooth two-dimensional vortex to axisymmetry after the application of an instantaneous, weak external strain field. In this limit the disturbance decays exponentially in time at a rate that is linked to a pole of the associated linear inviscid problem (known as a Landau pole). As a model of a typical vortex distribution that can give rise to cat's eyes, here distributions are examined that have a basic Gaussian shape but whose profiles have been artificially flattened about some radius $r_{c}$. A numerical study of the Landau poles for this family of vortices shows that as $r_{c}$ is varied so the decay rate of the disturbance moves smoothly between poles as the decay rates of two Landau poles cross. Cat's eyes that occur in the nonlinear evolution of a vortex lead to an axisymmetric azimuthally averaged profile with an annulus of approximately uniform vorticity, rather like the artificially flattened profiles investigated. Based on the stability of such profiles it is found that finite thickness cat's eyes can persist (i.e., the mean profile has a neutral mode) at two distinct radii, and in the limit of a thin flattened region the result that vanishingly thin cat's eyes only persist at a single radius is recovered. The decay of nonaxisymmetric perturbations to these flattened profiles for larger times is investigated and a comparison made with the result for a Gaussian profile. (C) 2008 American Institute of Physics. [DOI: 10.1063/1.2838596]
\end{abstract}

\section{INTRODUCTION}

Fluid flows at high Reynolds numbers can be dominated by the dynamics and interactions of long-lived vortical structures. ${ }^{1-4}$ The dynamics and stability of these vortices are particularly important in areas of fluid dynamics such as meteorology ${ }^{5,6}$ and magnetohydrodynamics. ${ }^{7}$ Many studies have considered the behavior of an axisymmetric vortex subjected to a transient, nonaxisymmetric strain. If this strain is weak then in the early stages the vortex may evolve dynamically into an axisymmetric state; any nonaxisymmetric components of the vorticity become finely scaled through spiral wind-up in the underlying flow field. ${ }^{8}$ During this process, the far-field form of the stream function decays exponentially in time and rotates with a fixed angular velocity which is fast compared to the decay rate. This behavior of the vortex is termed a quasimode $e^{9-11}$ and has been observed both in plasma experiments ${ }^{10}$ (where the governing equations are isomorphic to the Euler equations) and in numerical studies. $^{12}$

Put simply, a quasimode is essentially a wave located in the core of a vortex and, while it appears to be a single mode, it can more accurately be characterized as a wave-packet of continuum modes. The quasimode is a solution of the linearized Euler equations that is not separable in time, and decays on a much longer time-scale than the turn-over time-scale of the underlying vortex. Quasimodes of an axisymmetric circular vortex have been studied analytically via Laplace trans-

\footnotetext{
${ }^{a)}$ Author to whom correspondence should be addressed. Electronic mail: M.R.Turner@ex.ac.uk. Telephone: +441392 725280. Fax: +441392 217965 .
}

forms of the linearized Euler equation; ${ }^{9,10,13}$ an attraction of this method is that the angular velocity and the decay rate of the disturbance stream function can be deduced from the real and imaginary parts of a simple pole of the governing system. The location of this pole, known as a Landau pole, can be calculated by analytic continuation techniques ${ }^{9}$ and it follows that the decay rate and angular velocity of the quasimode depend only on the form of the axisymmetric base profile of the vortex. Moreover, the decay rate of the quasimode is very sensitive to the gradient of the axisymmetric base profile at the critical radius where the angular velocity of the quasimode equals that of the vortex. ${ }^{11}$

An illustration of an azimuthal wave number $n=2$ disturbance to a Gaussian vortex is shown in Fig. 1. At the earlier time [Fig. 1(a)] there is a linear combination of a spiral structure in which vorticity depends on $r$ and $\theta$, and a mode in which the sign of the vorticity depends just on $\theta$ : this is the quasimode, and corresponds to an elliptical distortion of the whole vortex that rotates (much like a Kelvin mode on a top-hat vortex) and decays because of interaction with spiral wind-up of fluctuations at the critical radius where the fluid corotates with the mode. At the later time [Fig. 1(b)] the quasimode has decayed to low levels, leaving behind the spiral structure, in which vorticity is of fine scale and essentially passive.

When an axisymmetric vortex is placed within a sufficiently strong irrotational strain field, it is well known that the vorticity can evolve into a tripole in which a pair of cat's eyes rotate around a coherent vortex core. ${ }^{10,11,14,15}$ The vorticity within the cat's eyes becomes wound up and homogenized; moreover, if cat's eyes persist (i.e., the far-field 


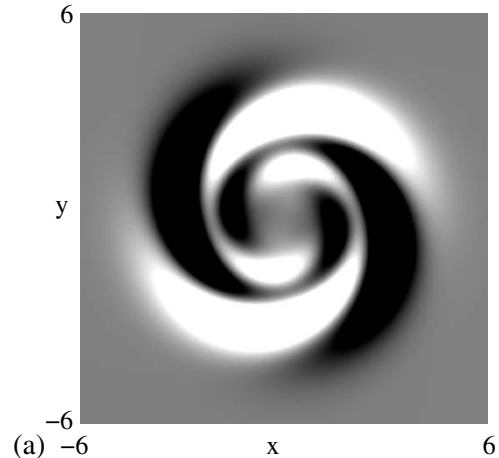

6

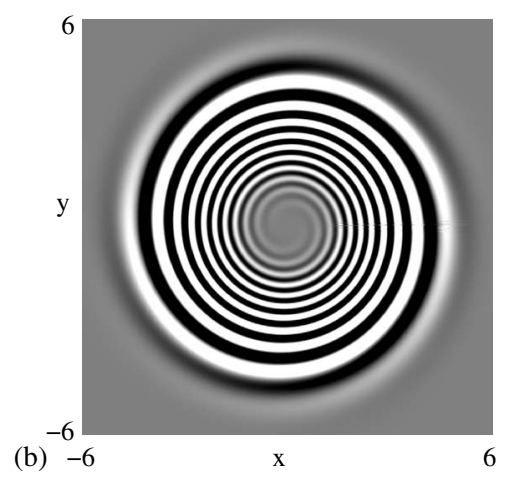

FIG. 1. An $n=2$ disturbance to a Gaussian vortex at (a) $t=200$ and (b) $t=1000$. The scale is white/black, where the vorticity is greater/less than 0.5 times the maximum/minimum vorticity. stream function neither grows nor decays) then they can be associated with a neutral mode of the mean vortex profile. On the other hand, if they are not maintained, decay occurs at a rate that corresponds to a Landau pole. ${ }^{15}$ Numerical simulations of the Navier-Stokes equations by Turner and Gilbert $^{15}$ show how finite thickness cat's eyes can be generated by forcing an axisymmetric vortex with a rotating strain field. When the strain field is switched on, cat's eyes form around the radius where the angular velocity of the vortex equals that of the strain field. If the strain field is of high amplitude or left on for a long duration, then when it is turned off, cat's eyes may persist at a radius whose position depends upon how the perturbation vorticity feeds back to change the axisymmetric basic profile. ${ }^{15}$ In contrast, some earlier studies ${ }^{16}$ have indicated that when cat's eyes generated on a Gaussian vortex are vanishingly thin, they are localized at a single specific radius. The full simulations ${ }^{15}$ could not access this limit of infinitesimal thickness, as unfeasibly long runs at very small amplitudes and very high Reynolds numbers would have been required.

The purpose of the current work is to ascertain whether finite thickness cat's eyes on a Gaussian vortex can be maintained at many radii or whether they are restricted to a single specific radius. Can these cat's eye structures superimposed on the vortex be long lived and, if so, what are the key properties of the underlying axisymmetric profile that enable it to support a neutral mode? We shall examine these issues by calculating the Landau poles of a model axisymmetric profile with a small flattened region or "defect." Our chosen profile [detailed in Eq. (18) below] comprises a standard Gaussian form supplemented by a three-parameter family of distributions; in essence the three parameters control the amplitude, thickness, and location of the defect. This form of vorticity distribution is quite typical of the azimuthal average of a vortex that can sustain a cat's eyes structure through vorticity homogenization in the eyes.

The present paper is laid out in the following way. In Sec. II we formulate the Landau pole problem and then in Sec. III describe how the poles move in the complex plane as the three parameters of the defect are varied. The Landau poles are tracked by solving a suitable eigenvalue problem formulated in Laplace transform space. The results obtained suggest that a profile with a thin homogenized layer of vorticity can support a neutral mode and, in the limit of a vanishingly thin layer, the position of the neutral mode is as predicted by the asymptotic work of Le Dizès. ${ }^{16}$ We also examine the large-time behavior of the far-field stream function and achieve this by solving the linearized Euler equation using a Keller box method. ${ }^{17}$ Some concluding remarks are made in Sec. IV.

\section{FORMULATION OF THE LANDAU POLE PROBLEM}

We consider a vortex governed by the two-dimensional, incompressible Euler equations in standard plane polar coordinates $(r, \theta)$,

$$
\begin{aligned}
& \partial_{t} \Omega-r^{-1}\left(\partial_{r} \Psi \partial_{\theta} \Omega-\partial_{\theta} \Psi \partial_{r} \Omega\right)=0, \\
& \nabla^{2} \Psi=-\Omega, \quad \nabla^{2} \equiv \partial_{r}^{2}+r^{-1} \partial_{r}+r^{-2} \partial_{\theta}^{2} .
\end{aligned}
$$

In this system, $\Omega(r, \theta, t)$ denotes the vorticity and $\Psi(r, \theta, t)$ is the corresponding stream function, which can found by inverting Eq. (2) and is permitted to grow no faster than $\ln r$ for large $r$.

We decompose the vorticity and stream function distributions into an axisymmetric part and a weak nonaxisymmetric perturbation,

$$
\begin{aligned}
& \Omega=\Omega_{0}(r)+\hat{\epsilon} \omega_{n}(r, t) e^{i n \theta}+\text { c.c. }+O\left(\hat{\epsilon}^{2}\right), \\
& \Psi=\Psi_{0}(r)+\hat{\epsilon} \psi_{n}(r, t) e^{i n \theta}+\text { c.c. }+O\left(\hat{\epsilon}^{2}\right) ;
\end{aligned}
$$

here the azimuthal mode number $n \geqslant 1, \hat{\epsilon} \ll 1$, and "c.c." denotes the complex conjugate. In this limit, Eqs. (1) and (2) reduce to the linearized Euler equations for the perturbation quantities,

$$
\begin{aligned}
& \partial_{t} \omega_{n}+\operatorname{in} \alpha(r) \omega_{n}+\operatorname{in} \beta(r) \psi_{n}=0, \\
& \Delta \psi_{n}=-\omega_{n}, \quad \Delta \equiv \partial_{r}^{2}+r^{-1} \partial_{r}-r^{-2} n^{2} .
\end{aligned}
$$

The angular velocity $\alpha(r)$ and the quantity $\beta(r)$ are given by

$$
\alpha(r)=-r^{-1} \partial_{r} \Psi_{0}, \quad \beta(r)=r^{-1} \partial_{r} \Omega_{0},
$$

where the axisymmetric quantities are coupled via $\Omega_{0}=-r^{-1} \partial_{r}\left(r \partial_{r} \Psi_{0}\right)$.

The properties of Laplace transforms of Eq. (5) have been studied in depth by Briggs et al. ${ }^{9}$ and Schecter et al., ${ }^{10}$ so we only highlight the main points here. (Further details can be obtained from those papers and from Ref. 15.) The quantity $n$ that appears in the above equations identifies the particular Fourier mode under investigation; notice that 
$n=1$ is a special case of a pure translation, which gives no dynamical effects; hence, it is of no interest here. ${ }^{18,19}$ We shall take $n=2$ in all our subsequent calculations for two main reasons. First, and conveniently, this mode is next sequentially in a general multipole expansion after the $n=1$ mode. On more physically significant grounds, the multipole expansion of a single vortex in a large scale flow will be dominated by the $n=2$ component in the presence of long distance interactions with other vortices; for general $n$, the effect of one vortex on another falls off $\propto R^{-n}$, where $R$ is their separation, so that the largest dynamical effect corresponds to $n=2$. We would also expect qualitatively similar results to those described here for the minority of flows for which the $n=2$ mode is completely absent so that the multipole moment with some other $n>2$ dominates the dynamical effects internal to the vortex. Notice that although our calculations will be restricted to the $n=2$ case, we shall retain a general $n$ in our formulation to illustrate the role of the mode number in the structure of the final equations.

We define the Laplace transform pair with respect to $t$ by

$$
\begin{aligned}
& \bar{f}(p)=\int_{0}^{\infty} e^{i p t} f(t) d t \\
& f(t)=-\frac{1}{2 \pi} \int_{\infty+i \sigma}^{-\infty+i \sigma} e^{-i p t} \bar{f}(p) d p,
\end{aligned}
$$

with any real $\sigma>0$. Combining Eqs. (5) and (6) and taking the Laplace transform with respect to $t$ leads to an equation for $\bar{\psi}_{n}(r, p)$ of the form

$$
\left[\frac{\partial^{2}}{\partial r^{2}}+\frac{1}{r} \frac{\partial}{\partial r}-\frac{n^{2}}{r^{2}}+\frac{n \beta(r)}{p-n \alpha(r)}\right] \bar{\psi}_{n}(r, p)=-\frac{i \omega_{n}(r, 0)}{p-n \alpha(r)} .
$$

If the $n$th multipole moment $Q_{n}(t)$ is defined by

$$
Q_{n}=\int_{0}^{\infty} r^{n+1} \omega_{n}(r, t) d r \quad(n \geqslant 1),
$$

then the evolution of $\ln \left|\operatorname{Re}\left(Q_{2}\right)\right|$ for a Gaussian vortex acted upon by a weak instantaneous strain ${ }^{12}$ is shown in Fig. 2 . Three distinct regimes of decay are apparent. Regime A is the quasimode period of exponential decay with decay rate $\gamma_{\mathrm{G}} \approx-0.0063$ and angular velocity $\alpha_{\mathrm{G}} \approx 0.0089$ calculated via Landau pole methods, ${ }^{15}$ while regimes $\mathrm{B}$ and $\mathrm{C}$ are related to the large- $r$ form of the vorticity and the remnant of the vorticity in the core, respectively. ${ }^{12}$ Schecter et al. ${ }^{10}$ give the quasimode decay rate as $(0.226-0.079 i) \Omega_{0}(0)$, where we have taken $\Omega_{0}(0)=1 / 4 \pi$. The minor difference between the angular velocity values of Turner and Gilbert ${ }^{15}$ and Schecter et $a l^{10}$ can be traced to the fact that the former was calculated using an infinite domain and the latter a large finite domain.

The multipole moment can be written in terms of the far-field form of the stream function by

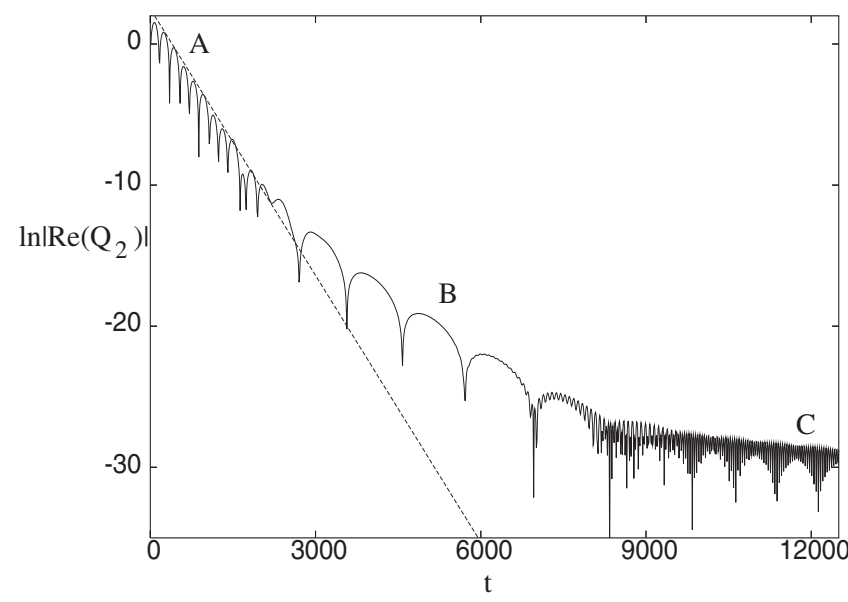

FIG. 2. Plot of $\ln \left|\operatorname{Re}\left(Q_{2}\right)\right|$ for a Gaussian vortex as a function of $t$. The dashed line gives the regime A decay rate $\gamma_{\mathrm{G}}=-0.0063$ from a Landau pole calculation.

$$
Q_{n}(t)=\lim _{r \rightarrow \infty}\left(n r^{n} \psi_{n}-r^{n+1} \partial_{r} \psi_{n}\right)
$$

(see Ref. 12). We impose the boundary condition $\psi_{n}=0$ at $r=r_{0}$ where we will take the limit $r_{0} \rightarrow \infty$, so as to simulate the evolution of a disturbance in an infinite domain.

Equation (10) is solved by means of the appropriate Green's function ${ }^{9,13}$ that leads to the Laplace transform of the quantity $Q_{n}(t)$ expressed as

$$
\bar{Q}_{n}(p)=-\left.\frac{i r^{n+1}}{\Psi_{\mathrm{L}}(r, p)} \int_{0}^{r} \frac{s \Psi_{\mathrm{L}}(s, p) \omega_{n}(s, 0)}{r(n \alpha(s)-p)} d s\right|_{r=r_{0}},
$$

where the function $\Psi_{\mathrm{L}}(r, p)$ satisfies the homogeneous form of Eq. (10),

$$
\left[\frac{\partial^{2}}{\partial r^{2}}+\frac{1}{r} \frac{\partial}{\partial r}-\frac{n^{2}}{r^{2}}+\frac{n \beta(r)}{p-n \alpha(r)}\right] \Psi_{\mathrm{L}}(r, p)=0,
$$

subject to the condition $\Psi_{\mathrm{L}}(0, p)=0$.

It is clear that Eq. (13) potentially has a singularity at the point $r_{s}$, where $\alpha\left(r_{s}\right)=p / n$. If the axisymmetric vorticity profile $\Omega_{0}(r)$ is a smooth decreasing function and if $\beta\left(r_{s}\right)$ vanishes, then it can be shown that there is a neutral mode of the vortex. ${ }^{9}$ Conversely, if $\beta\left(r_{s}\right) \neq 0$ and $\Omega_{0}(r)$ is monotonically decreasing on the whole domain $r \in\left[0, r_{0}\right]$, then there are no discrete eigenmodes of the vortex. ${ }^{9}$ In the first case the function $\bar{Q}_{n}(p)$ possesses a simple pole, which on inverting the Laplace transform gives rise to an exponential term in $Q_{n}(t)$. In the second case, however, we have no simple poles of $\bar{Q}_{n}(p)$ and hence no exponential terms in $Q_{n}(t)$. All the behavior of $Q_{n}(t)$ is contained in the branch cut along the real $p$-axis in the range $n \alpha\left(r_{0}\right) \leqslant p \leqslant n \alpha(0)$. Analytical continuation techniques enable this branch cut to be deformed below the real $p$-axis by moving the radial contour of integration in Eq. (12) above the real $r$-axis. If the branch cut is bent sufficiently, then one or more so-called Landau poles $p_{j}$ $(j=1,2,3, \ldots)$ appear in the analytical continuation of $\bar{Q}_{n}(p)$ [the number of poles that are seen depends both on how far the contour is deformed and the exact form of $\Omega_{0}(r)$ ]. When the inversion contour is deformed around a pole and the 

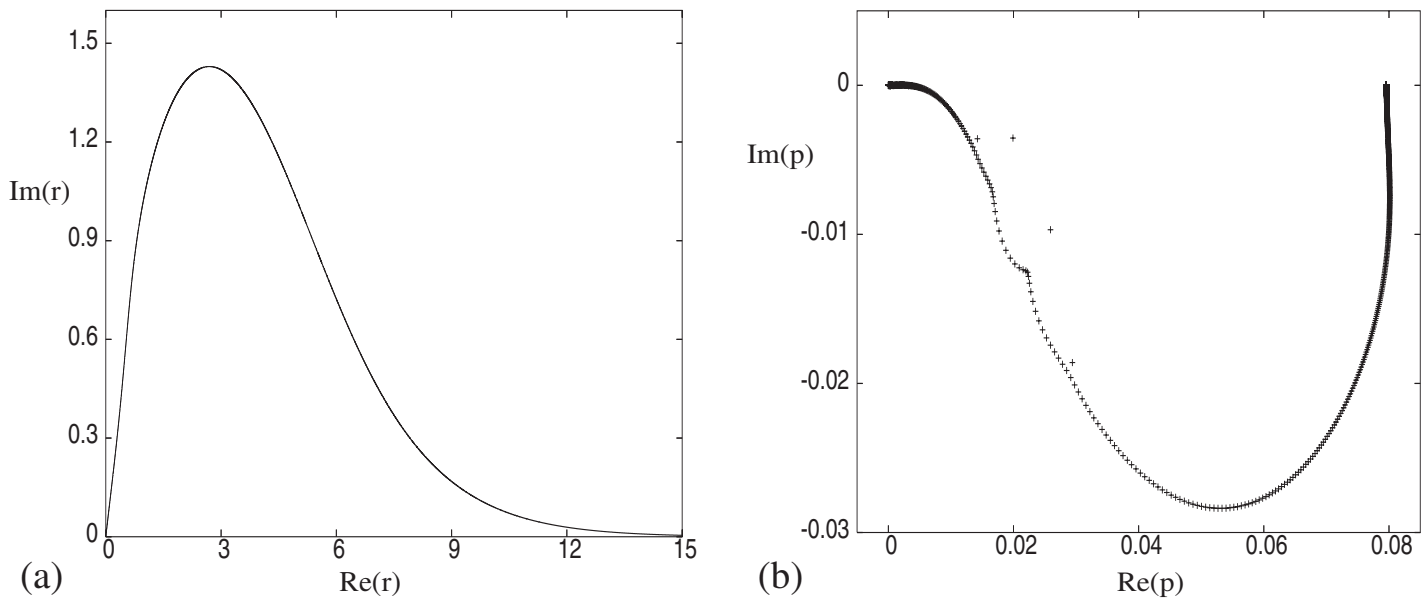

FIG. 3. Plot of (a) the contour in the complex $r$-plane given by Eq. (16) with $\rho_{1}=1.7$ and $\rho_{2}=2$ and (b) a discrete approximation to the continuous spectrum and four Landau poles in the complex $p$-plane for an axisymmetric vorticity profile (18), with $\delta=0.1, \epsilon=0.5$, and $r_{c}=4$.

branch cut, this leads to a term in $Q_{n}(t)$ of the form $e^{-i p_{j} t}$; although such a term can dominate the early evolution of $Q_{n}(t)$, it does not correspond to a normal mode. This Landau pole identifies a complex decay rate $-i p_{j}=\gamma_{Q}-i n \alpha_{Q}$, where $\gamma_{Q}<0$ and $\alpha_{Q}$ are the decay rate and angular velocity, respectively. When there are $N$ Landau poles in the analytical continuation of $\bar{Q}_{n}(p)$, then $Q_{n}(t)$ takes the form

$$
Q_{n}(t)=\sum_{j=1}^{N} f_{j} e^{-i p_{j} t}+\text { a branch cut contribution, }
$$

where the constants $f_{j}$ depend on the initial conditions. The angular velocity $\alpha_{Q}$ of each quasimode identifies a corresponding critical radius $r_{Q}$ at which the fluid particles corotate. This radius is found by solving

$$
\alpha\left(r_{Q}\right)=\alpha_{Q},
$$

where we have used Eq. (7) to write $\alpha(r)$ as

$$
\alpha(r)=\frac{1}{r^{2}} \int_{0}^{r} s \Omega_{0}(s) d s .
$$

For a pure Gaussian vortex $\alpha_{\mathrm{G}} \approx 0.0089$, which gives $r_{\mathrm{G}} \approx 4.22$. With the vortex profiles we consider in this study, small $\alpha_{Q}$ corresponds to a large critical radius $r_{Q}$, while if $\alpha_{Q} \approx \alpha(0)$, then $r_{Q} \approx 0$. With a linear combination of contributions such as in Eq. (14) it is not clear just how $Q_{n}(t)$ might behave during its early evolution, as the relative values of the quantities $f_{j}$ are important. Nevertheless, after a sufficiently long initial transient, it will be the Landau pole with smallest decay rate that will eventually dominate.

To calculate the positions of the Landau poles we used contours in the complex $r$-plane, parametrized by $s$, of the general form

$$
r(s)=\tan \left\{\frac{\pi}{2}\left[s+i \rho_{1} s \exp \left(\frac{1}{\rho_{2}(s-1)}\right)\right]\right\},
$$

where $\rho_{1}$ and $\rho_{2}$ are real constants. As $s$ moves from 0 to 1 so the corresponding $r$-path joins zero to (real) infinity and a typical form of the contour is sketched in Fig. 3(a).
Equation (13) is integrated from $s=\widetilde{s} \ll 1$ to $s=1$ along Eq. (16) using a Runge-Kutta method. As $r \rightarrow 0$, it is known that $\Psi_{\mathrm{L}}(r, p) \sim r^{n}$, and thus we impose the initial conditions

$$
\Psi_{\mathrm{L}}(r(\widetilde{s}), p)=r^{n}(\widetilde{s}), \quad \frac{\partial \Psi_{\mathrm{L}}}{\partial s}=n r^{\prime}(\widetilde{s}) r^{n-1}(\widetilde{s}),
$$

at $s=\widetilde{s} \ll 1$. Of course, in general $\Psi_{\mathrm{L}} \neq 0$ at $s=1$, and thus a Newton iteration on the eigenvalue $p$ is used to ensure that $\Psi_{\mathrm{L}}$ vanishes at this endpoint. An alternative strategy for solving Eq. (13) subject to homogeneous boundary conditions relies on a global eigenvalue method ${ }^{20}$ that generates a discrete approximation to the continuous spectrum as well as finding any poles around which the inversion contour has to be deformed. The global method is used to give an initial starting value to the eigenvalue $p$ for the iterative local method, which is generally the more accurate. An example of the global solution to Eq. (13) is shown in Fig. 3(b), which illustrates the continuous spectrum together with four Landau poles.

Bearing in mind our objective to investigate when a cat's eyes structure might be relatively long lived, we consider the three-parameter family of axisymmetric profiles given by

$$
\begin{aligned}
\Omega_{0}\left(r ; \delta, \epsilon, r_{c}\right)= & \frac{1}{4 \pi} e^{-r^{2} / 4}+\delta \frac{r_{c}\left(r-r_{c}\right)}{8 \pi} \\
& \times \exp \left(\frac{r}{4 r_{c}}\left(2-r_{c}^{2}\right)-\frac{1}{2}-\frac{\left(r-r_{c}\right)^{2}}{\epsilon^{2}}\right) .
\end{aligned}
$$

Here, $\delta, \epsilon$, and $r_{c}$ denote the amplitude, thickness, and radius of the defect, respectively. Some sample profiles are shown in Fig. 4, which illustrates the different forms of $\Omega_{0}(r)$ as the three parameters are varied. The form of Eq. (18) is that of a Gaussian vortex that has been flattened off in the vicinity of the critical $r_{c}$; as mentioned earlier, this type of profile is characteristic of those for which cat's eyes are observed. The parameter $\delta$ is a measure of the degree of flattening imposed; clearly, when $\delta=0$, the Gaussian is unaltered and at the other extreme $(\delta=1)$, there is a turning point and an inflection point at $r=r_{c}\left[\Omega_{0}^{\prime}\left(r_{c}\right)=\Omega_{0}^{\prime \prime}\left(r_{c}\right)=0\right]$, which we shall refer to as 


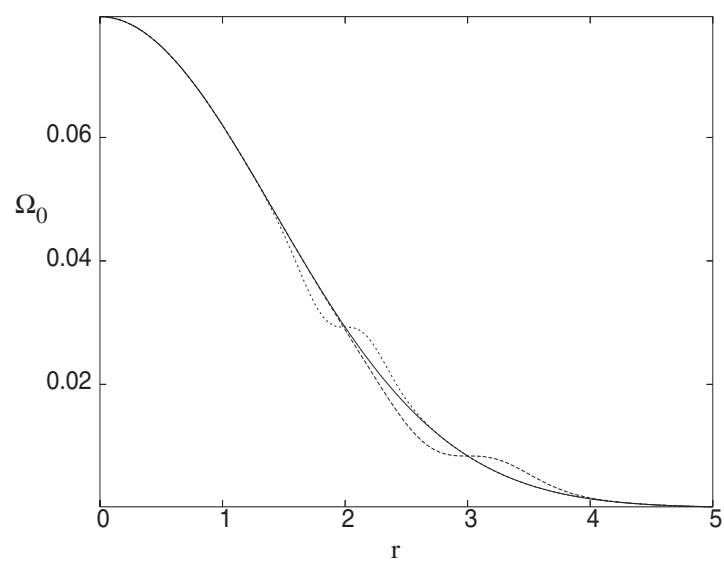

FIG. 4. Figure showing $\Omega_{0}(r)$ for $\delta=0$ (solid line); $\delta=1, \epsilon=0.5, r_{c}=3$ (dashed line), and $\delta=1, \epsilon=0.3, r_{c}=2$ (dotted line).

a "flat region" for brevity. This zone can be thought of as the azimuthal average of a vortex that has cat's eyes at a radius $r_{c}$ of thickness $\epsilon$, which have been formed through some nonlinear mechanism. However, vortices containing cat's eyes, which have been generated in fully nonlinear simulations, do not have a smooth azimuthal profile ${ }^{15}$ but, instead, typically contain a flattened region with fine scale structure due to the nonlinear interactions. They also generally have sharper vorticity gradients around the flattened region than are present in the idealized profile [Eq. (18)]. Thus, our model used here might be helpful for understanding the behaviors of thin cat's eyes, but nonlinear interactions need to be considered for our results to be confirmed.

\section{RESULTS}

In this section we describe results of the Landau pole calculations and the linear code simulations for the profiles given by Eq. (18). We shall have frequent cause to refer to the real and imaginary parts of Landau poles $p_{j}$ and thus it is to be remembered that these quantities correspond to $n$ multiples of the angular velocity $\alpha_{Q}$ and the decay rate $\gamma_{Q}$ of the vortex quasimode, respectively.

\section{A. Landau pole calculations}

The Landau pole calculations rely on the parameters $\rho_{1}$ and $\rho_{2}$ in Eq. (16) being chosen so that the corresponding contour of the continuous spectrum in the $p$-plane is bent sufficiently to reveal the poles we wish to follow. All the results here used $\rho_{1} \in[1.5,5]$ and $\rho_{2} \in[0.4,2.2]$ with the particular values governed by the nature of the underlying vorticity $\Omega_{0}(r)$. In the limits of small $\epsilon$ and extreme $r_{c}$ (either small or large), we found that it was not always possible to track all the poles of interest as the contour in the $p$-plane often becomes very knotted and intricate. Moreover, the poles then become easily confused with the continuous spectrum. That said, however, we were able to capture the behavior of the poles sufficiently well to make some useful observations.

We have a three-dimensional parameter space $\left(\delta, \epsilon, r_{c}\right)$ to investigate and for the parameter ranges considered, we generally found that the values of $\rho_{1}$ and $\rho_{2}$ in Eq. (16) led to three Landau poles of interest in the $p$-plane; the only exception occurs when $\delta=1$ when an extra pole appears. We explored our parameter space systematically by fixing the parameters two at a time and then allowing the third to vary. Figure 5 plots the imaginary parts $\operatorname{Im}(p)=\gamma_{Q}$ of three Landau poles as the basic vorticity changes.

Figure 5(a) relates to a defect of moderate thickness $\epsilon=0.5$ positioned at $r_{c}=4$, whose amplitude $0 \leqslant \delta \leqslant 1$. For $\delta=0$ we have a Gaussian profile, which has a Landau pole at $p_{\mathrm{G}}=0.0177-0.0063 i$, which corresponds to pole 1 . As $\delta$ is increased from zero, so poles 2 and 3 move in quickly from the large negative imaginary region of the $p$-plane. One of the poles (pole 3 ) has a larger decay rate than the other two for all the values of $\delta$ considered and hence is expected to be insignificant for the initial decay of the perturbation. Poles 1 and 2 have very similar decay rates for $0<\delta<0.2$, so it is unclear at which rate the vortex quasimode will decay in this region. As we approach the case of a flat region at $r=r_{c}$ in $\Omega_{0}(r)$ (i.e., the amplitude of the defect $\delta=1$ ), then we find that the original Gaussian quasimode Landau pole (pole 1) again has the smallest decay rate, and hence should dominate the initial decay of the perturbation.

In Fig. 5(b) the defect has a small fixed amplitude $\delta=0.1$ and is positioned at the point $r_{c}=4$, but has a variable thickness $\epsilon$. The two Landau poles that had similar decay rates in Fig. 5(a) continue to do so over the whole range of $\epsilon$ investigated. Pole 3 is again insignificant over most of the $\epsilon$-domain as it has a much larger decay rate than the other two, although, as $\epsilon$ becomes smaller (i.e., as the defect thins), the presence of this third pole becomes more important.

Lastly, the defect in Fig. 5(c) has a fixed small amplitude $(\delta=0.1)$ and is of moderate thickness $(\epsilon=0.5)$, but is moved around by varying $r_{c}$. Close to $r_{c}=4$, the two poles, which were almost coincident in Fig. 5(a), remain near to each other, and actually swap places. We would therefore expect the decay rate of the perturbation to change between these two values with a corresponding jump in frequency (cf. Fig. 10 later). Pole 3 again remains insignificant for the values of $r_{c}$ considered here; however, for large $r_{c}$ (i.e., when the defect is positioned well away from the vortex core) this pole tends to the Landau pole value for a Gaussian vortex noted earlier. As $r_{c}$ is moved towards the origin, with $\epsilon$ still moderately large, the core of the vortex becomes increasingly deformed and sharp gradients of vorticity form around the defect. Consequently, results with $r_{c}<1$ and moderate $\epsilon$ need to be treated with care although if the thickness of the defect $\epsilon$ is reduced as $r_{c}$ moves towards the origin then results for smaller values of $r_{c}$ can be attained.

Figure 5(a) suggests that when there is a flat region in the profile $(\delta=1)$, there appears to be a single Landau pole which dominates the decay of the perturbation. This is examined further in Fig. 6, which shows the real and imaginary parts of the significant Landau poles as functions of the defect position $r_{c}$ for amplitude $\delta=1$ and thickness $\epsilon=0.5$. We see that there are now four Landau poles which appear in the region of the $p$-plane we consider. Figure 6(b) shows that poles 3 and 4 are not important in the evolution of the perturbation, as their decay rates are larger than the other two. 

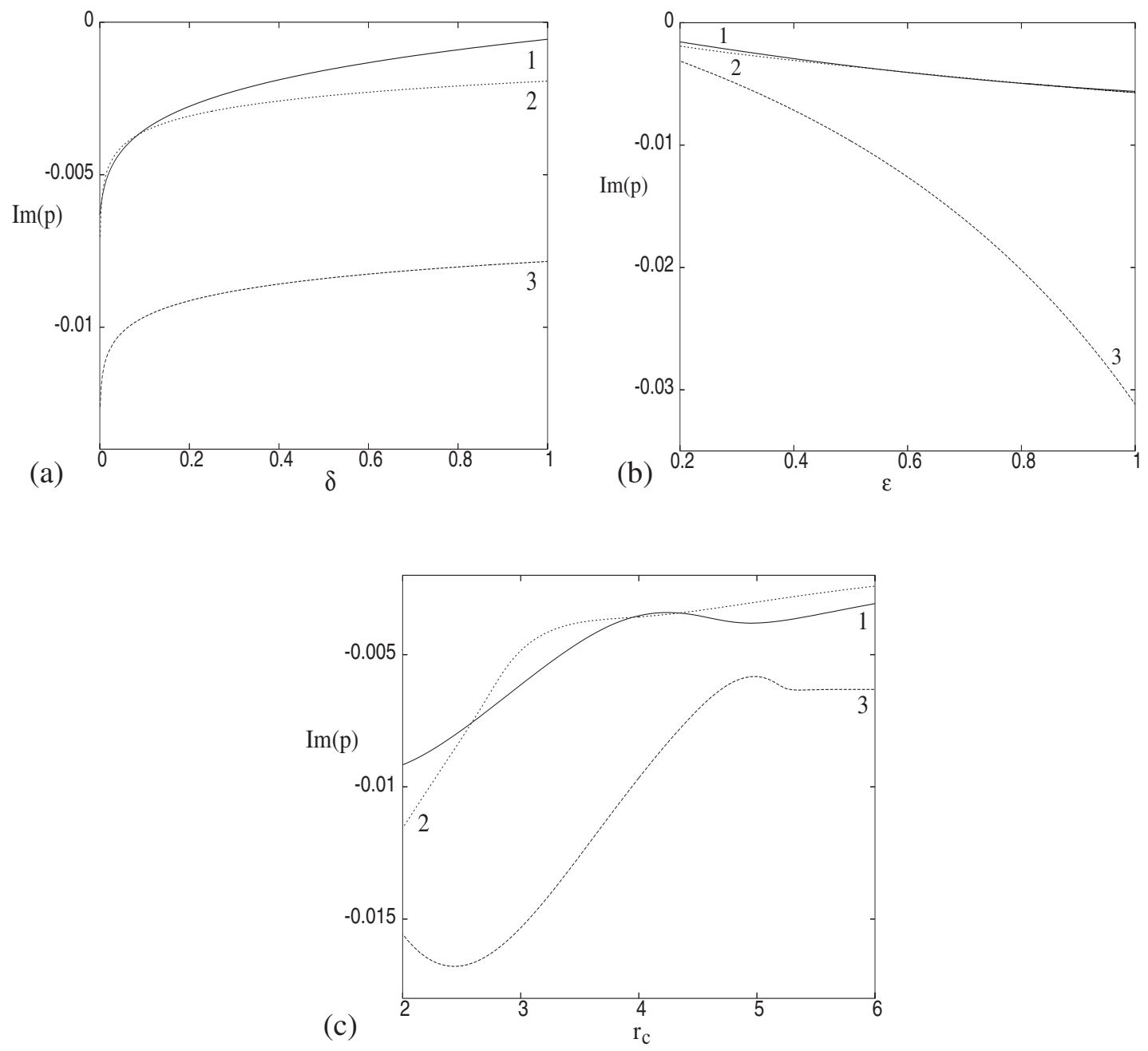

FIG. 5. Plot of the imaginary parts of three Landau poles as a function of (a) $\delta$ for $\left(\epsilon, r_{c}\right)=(0.5,4)$, (b) $\epsilon$ for $\left(\delta, r_{c}\right)=(0.1,4)$, and (c) $r_{c}$ for $(\delta, \epsilon)=(0.1,0.5)$.

When $r_{c}>4.5$, there are two poles close together, and thus we expect these to combine together to affect the decay rate of perturbations to the vortex. For $1<r_{c}<4.5$, there is just one pole that dominates the decay of the vortex perturbation and it is this pole on which we concentrate now. This pole is significant because at $r_{c} \approx 2.6$, the vortex has a decay rate of only $\gamma_{Q} \approx-6.4 \times 10^{-5}$, and thus at this location the vortex perturbation will decay extremely slowly. Indeed, this decay
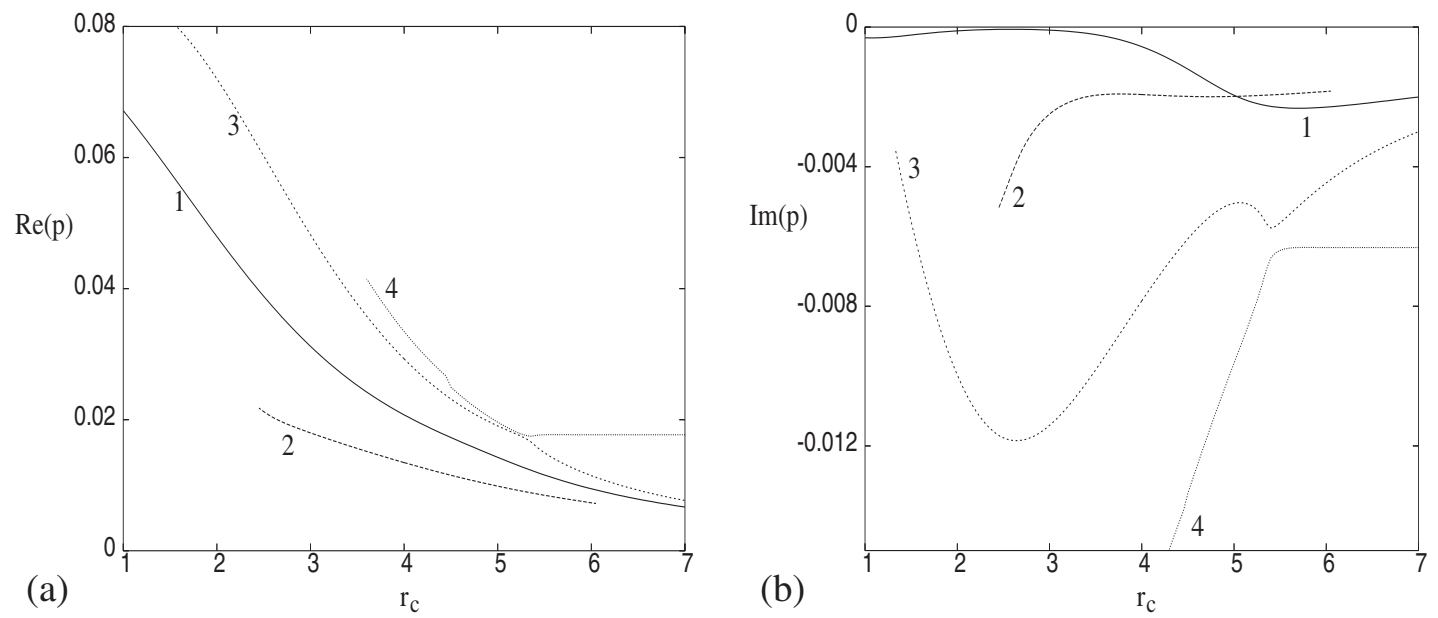

FIG. 6. Plot of (a) the frequency or real part $\operatorname{Re}\left(p_{j}\right)=n \alpha_{Q}$ and (b) the decay rate or imaginary part $\operatorname{Im}\left(p_{j}\right)=\gamma_{Q}$ of four Landau poles as a function of $r_{c}$ for $\delta=1.0$ and $\epsilon=0.5$. 


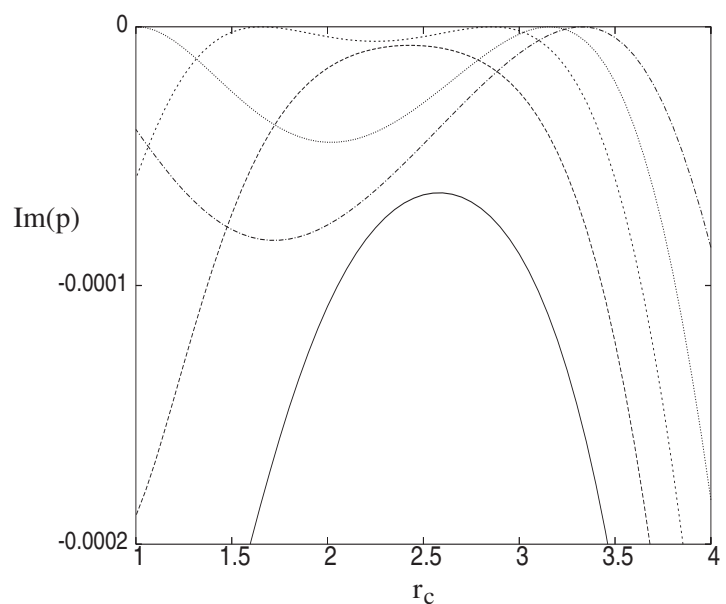

FIG. 7. Figure showing the decay rate $\gamma_{Q}=\operatorname{Im}(p)$ for the Landau pole with the smallest decay rate as a function of $r_{c}$ with $\delta=1$ and $\epsilon=0.5$ (solid line), 0.4 (large dashed line), 0.3 (small dashed line), 0.2 (dotted line), and 0.1 (dot-dashed line), from the middle to top right.

is so slow that it suggests that only tiny changes to the details of the defect might be sufficient to ensure that $\gamma_{Q}=0$, thereby forming a neutral mode.

Figure 7 illustrates $\operatorname{Im}(p)=\gamma_{Q}$ for the Landau pole with the smallest decay rate, as a function of defect position $r_{c}$, for $\delta=1$ and various values of $\epsilon$. We see that as the defect thickness $\epsilon$ decreases from $\epsilon=0.5$ (solid line) the maximum growth rate value increases. However, when $\epsilon=0.3$ (dotted line) we see that there are now two maxima in the growth rate curve and these both have $\gamma_{Q}=0$; i.e., they are both neutral modes of the vortex. As $\epsilon$ is reduced further, both the neutral modes remain, but their positions separate. This suggests that cat's eyes of a given finite thickness $\epsilon$, which have an azimuthal average profile that contains an annulus of homogenized vorticity, can persist at two distinct radii. As $\epsilon \rightarrow 0$ we expect to recover the result of Le Dizès, ${ }^{16}$ who showed that vanishingly thin cat's eyes can only possess a neutral mode at the one radius $r_{\mathrm{LD}}=3.44$ (using our scalings).

Figure 8 (a) plots the value of $r_{c}$ at which the maximum value of $\operatorname{Im}(p)=\gamma_{Q}$ occurs as a function of the defect thick-

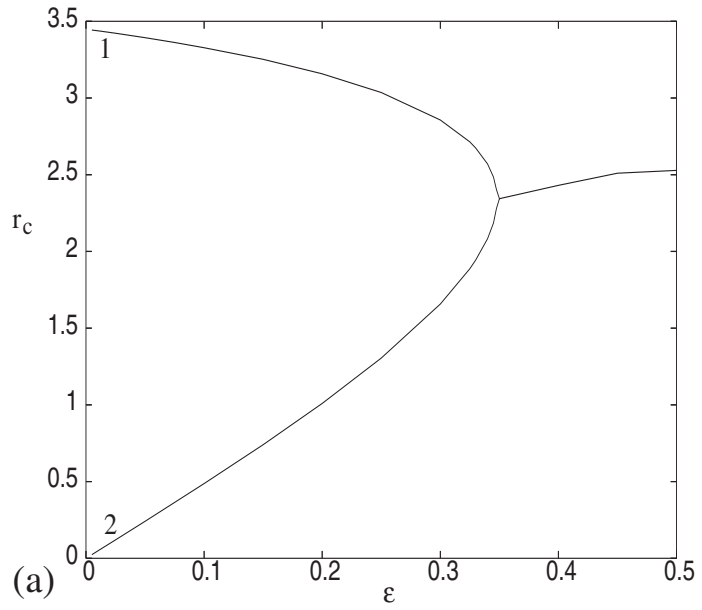

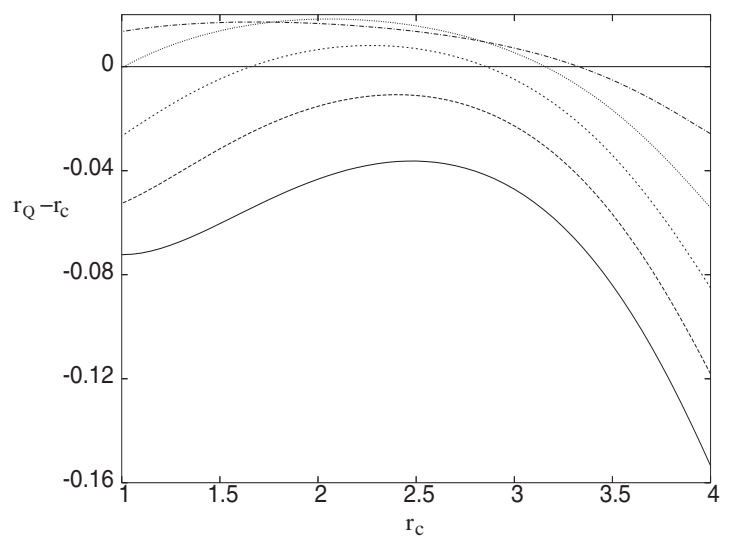

FIG. 9. Plot of $r_{Q}-r_{c}$ against $r_{c}$ for $\delta=1$ and the values of $\epsilon$ and the line styles from Fig. 7 with $\epsilon=0.5$ at the bottom to $\epsilon=0.1$ at the top. The solid horizontal line represents the line $r_{Q}=r_{c}$.

ness $\epsilon$ (with $\delta=1$ ). When $\epsilon \lesssim 0.35$, there are two positions at which the maximum $\gamma_{Q}$ occurs; at these points $\gamma_{Q}$ is zero and thus these correspond to neutral modes. Figure 8(b) shows the dependence of $2 \alpha_{Q}=\operatorname{Re}(p)$, where the minimum value of $\gamma_{Q}$ occurs as a function of the thickness $\epsilon$. The neutral mode that moves towards the origin (branch 2) has an angular velocity that increases as $\epsilon$ is reduced, and in the limit $\epsilon \rightarrow 0$, so $\alpha_{Q} \rightarrow 1 / 4 \pi$. Le Dizés ${ }^{16}$ has shown that a vanishingly thin critical layer cannot exist at the origin, and we leave the issue of the limit $\epsilon \rightarrow 0$ along branch 2 as a topic for future study. Linear extrapolation suggests that the position and angular velocity of the branch 1 neutral mode tend to $r_{c}=3.448$ and $\alpha_{Q}=0.0127$ as $\epsilon \rightarrow 0$. Both of these results agree with the results $r_{\mathrm{LD}}=3.44$ and $\alpha_{\mathrm{LD}}=0.0127$ derived by Le Dizès ${ }^{16}$ with errors of less than $1 \%$.

An explanation for the occurrence of the two neutral modes seen in Figs. 7 and 8 is offered by Fig. 9. This shows the form of $r_{Q}-r_{c}$, found by solving Eq. (15), as a function of $r_{c}$ for $\delta=1$ and the five chosen defect thicknesses used in Fig. 7. We see that when $\epsilon=0.4$ or 0.5 , the critical radius $r_{c}$ always exceeds $r_{Q}$. This implies that the critical radius of the quasimode never occurs at the flat center of the defect in the

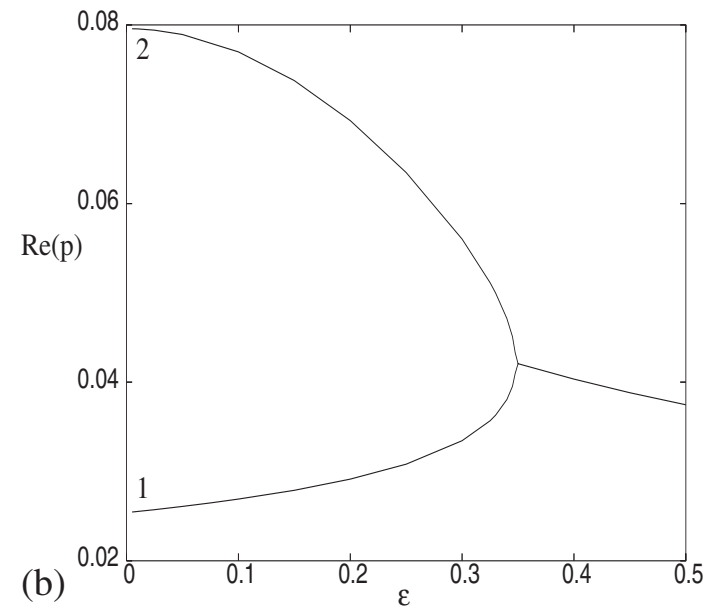

FIG. 8. Plot of (a) $r_{c}$ and (b) $\operatorname{Re}(p)=2 \alpha_{Q}$, where the maximum value of $\gamma_{Q}$ occurs as a function of $\epsilon$. The numbers indicate the two branches of solutions. 

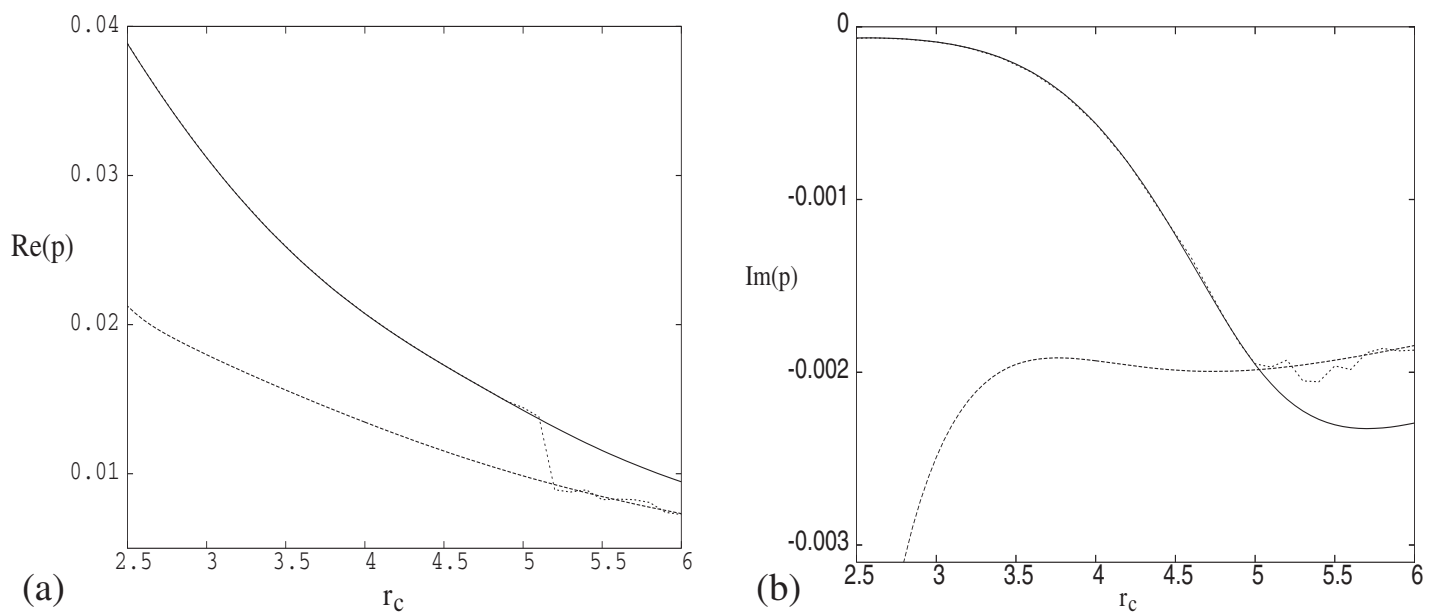

FIG. 10. Plot of (a) the real part and (b) the imaginary part of the growth rate calculated via the linear code (dotted line) as a function of $r_{c}$ for $\delta=1$ and $\epsilon=0.5$. The solid line and the large dashed line show the two Landau poles with largest growth rates in this region of interest. For $r_{c} \lesssim 5$, the solid and dotted lines are indistinguishable.

profile; in other words, the singular point $r_{Q}$ of Eq. (13) never occurs where $\beta=0$. On the other hand, the curves for other thicknesses have $r_{Q}=r_{c}$ twice at the two values of $r_{c}$ given in Fig. 8(a). Thus, the positions of the neutral modes correspond to points where the quasimode critical layer occurs exactly at the center of the flat region of the profile, i.e., the singular point of Eq. (13), $r=r_{Q}$, is where $\beta=0$. As the thickness $\epsilon$ of the flat region is reduced from 0.5 (reading up the curves), we find that the peak of the growth rate curve moves towards the line $r_{Q}=r_{c}$ and at $\epsilon \approx 0.35$, the curve and line become tangent to one another and there is a single neutral mode of the vortex. For values of $\epsilon \leq 0.35$, there are two neutral modes; one of these moves towards $r_{c}=3.448$ and the other approaches $r_{c}=0$ as the defect thickness $\epsilon \rightarrow 0$.

\section{B. Comparison with linear code}

Next we compare our Landau pole calculations with results determined by numerically solving Eq. (5) using a Keller box ${ }^{17}$ routine from the NAG suite; see Ref. 12 for more details. The scheme imposes an external flow which instantaneously distorts the vortex and then allows it to evolve freely over time. This problem is of initial value type and thus the decay rate and angular velocity measured from the solution of Eq. (5) are time dependent. To estimate the values $\gamma_{Q}$ and $\alpha_{Q}$, we took the best linear fit of the data between two time values that occur just before the vortex leaves the linear decay part of its evolution (i.e., just before the vortex leaves regime A in a case such as Fig. 2). These times were decided by a preliminary run of the code, from which we estimated the instant $t_{2}$ when the vortex changes regime. The earlier time was then taken to be $t_{1}=t_{2}-1000$, and then a re-run of the code gave decay rates and angular velocities that agree very well with the Landau pole theory.

The results outlined in Sec. III A show that in certain regions of our three-dimensional parameter space, one Landau pole dominates over the others, and hence we expect the multipole moment $Q_{2}(t)$ to decay exponentially with a decay rate and angular velocity given by this Landau pole. However, this is not completely obvious because this quasimode decay is just a transient (see regime A in Fig. 2) and thus there is only a finite time over which a decay rate can be measured before the contribution from the branch cut takes over. Hence, to assess at what rate the vortex perturbations actually decay in the early stages, we used the numerical scheme above to compare with our Landau pole results.

Figure 10 shows results from the solution to the linear equations (5) and (6) together with the Landau poles corresponding to the smallest decay rates for amplitude $\delta=1$ and thickness $\epsilon=0.5$ as in Fig. 6. Figure 10(a) demonstrates very good agreement between the predictions of the linear code and the real part of the Landau pole with the larger of the two angular velocities, at least for $2.5<r_{c}<5$. However, when the flat defect is centered at $r_{c} \approx 5$, a sudden change in the angular velocity of the perturbation occurs and the linear code results correlate with the Landau pole with the smaller of the two angular velocities once $r_{c}>5$. The reason for this frequency jump is suggested in Fig. 10(b), which shows the imaginary part of the Landau pole $\left(=\gamma_{Q}\right)$. The linear code gives a growth rate that agrees with the Landau pole with smallest decay rate for $2.5<r_{c}<5$; however, at $r_{c} \approx 5$ another Landau pole acquires a smaller decay rate, and thus the vortex perturbation switches onto this new rate, with the corresponding angular velocity given by this pole [see Fig. 10(a)]. The reason the two methods are not in complete agreement for $r_{c}>5$ is because when the decay rates of these two Landau poles become close, the vortex decays with a linear combination of the two decay rates and frequencies and distinguishing between them is not particularly easy.

Figure 11 considers the far-field amplitude $\ln \left|Q_{2}(t)\right|$ for a vorticity profile with amplitude $\delta=1$, thickness $\epsilon=0.5$, and defect position $r_{c}=5.2$ (solid line). This value of $r_{c}$ lies close to the point where the two growth rate curves meet in Fig. 6(b). The two straight lines represent two decay rates: the first given by the best fit analysis of the linear code (dashed line) and the second by the dominant Landau pole (dotted 


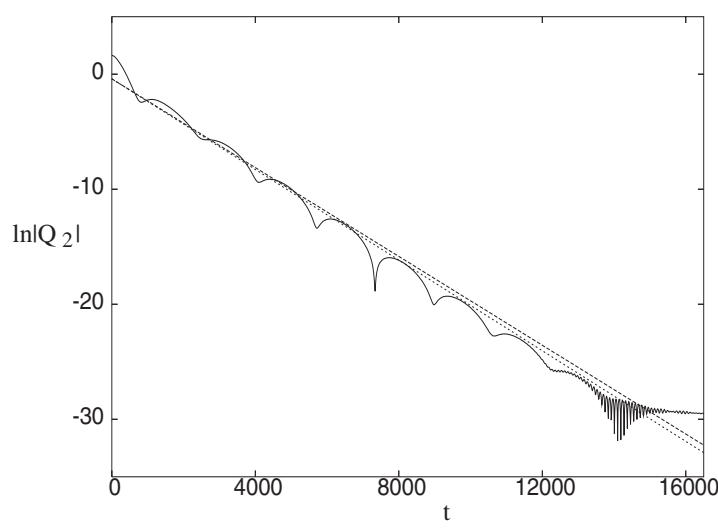

FIG. 11. Plot of $\ln \left|Q_{2}\right|$ against time for the case $\delta=1, \epsilon=0.5$, and $r_{c}=5.2$. The dashed line is the growth rate given by the linear code and the dotted line is the growth rate give by the dominant Landau pole.

line). We can see that due to the oscillations in $\ln \left|Q_{2}\right|$ a best fit line would depend on the time interval chosen in which to take the approximation and because this initial exponential decay is only a transient we cannot integrate until one Landau pole clearly dominates the solution; this is the reason for the small discrepancy between the results in Fig. 10 once $r_{c}>5$. The decay rate given by the Landau pole in Fig. 11 (dotted line) is in good agreement with the decay rate of $\ln \left|Q_{2}\right|$, and hence the vortex perturbation really does diminish at the rate corresponding to that Landau pole with the slowest decay rate.

The Landau pole calculation in Fig. 7 of Sec. III A shows that for defect thicknesses $\epsilon<0.35$, there are two positions $r_{c}$, where $\operatorname{Im}(p)=\gamma_{Q}=0$, and hence two radii that can support a neutral mode. We can confirm that a vortex does admit these two neutral modes by using the linear code for the basic profile with $\delta=1$ and $\epsilon=0.3$. The outcome is illustrated in Fig. 12; it is concluded that the linear code (dashed line) and the Landau pole result (solid line) are in excellent

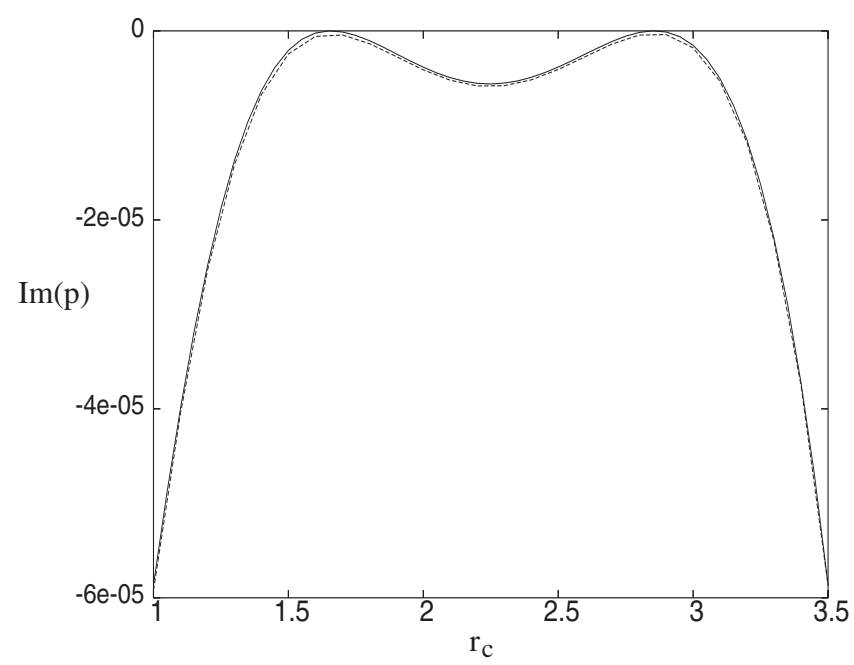

FIG. 12. Plot of $\operatorname{Im}(p)=\gamma_{Q}$ calculated by the linear code (dashed line) and the Landau pole code (solid line) as a function of $r_{c}$ for $\delta=1$, and $\epsilon=0.3$.

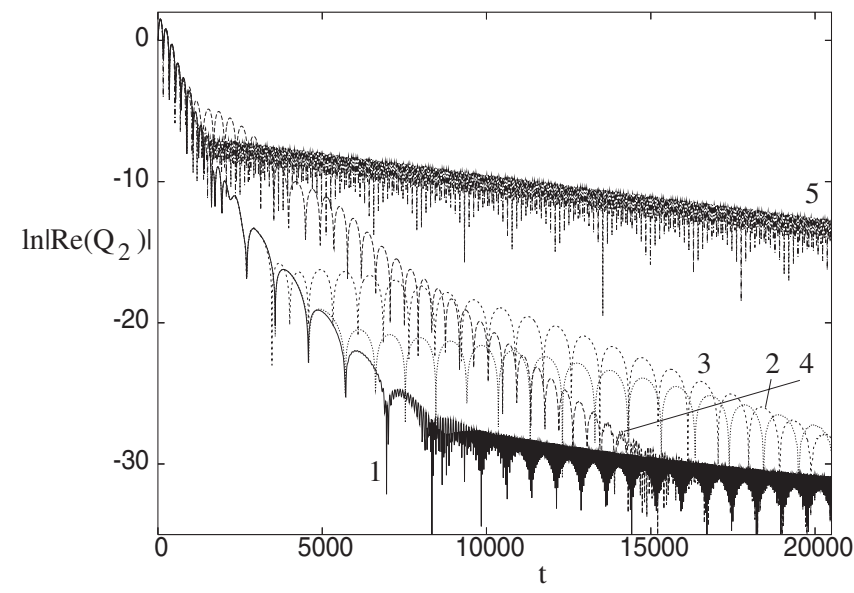

FIG. 13. Curve 1 shows a plot of $\ln \left|\operatorname{Re}\left[Q_{2}(t)\right]\right|$ when $\delta=0$. Curves $2-5$ all relate to the case when $\delta=1$ and $\epsilon=0.5$, but with $r_{c}=10,9,6$, and 1 , respectively.

agreement, and the frequencies $n \alpha_{Q}=\operatorname{Re}(p)$ from the two calculations (not shown) turn out to be indistinguishable over this range of $r_{c}$.

Figure 13 plots $\ln \left|\operatorname{Re}\left(Q_{2}(t)\right)\right|$ for the Gaussian vortex and four vorticity profiles, all with defect amplitude $\delta=1$ and thickness $\epsilon=0.5$, but with various positions in the range $1 \leqslant r_{c} \leqslant 10$. The Gaussian vortex (curve 1), discussed in Sec. II, has an initial period of exponential decay with $\gamma_{\mathrm{G}} \approx-0.0063$ followed by an interval of decay related to the large- $r$ form of the vorticity and finally a region of algebraic decay linked to the remnant of vorticity in the core of the vortex (see regimes B and C of Fig. 2). What makes Fig. 13 so interesting is that all the results with $\delta=1$ (curves 2-5) appear to decay at exactly the same rate and with the angular velocity of the Gaussian vortex (curve 1), at least up to some time. Thereafter, they start to decay exponentially at a rate determined by the dominant Landau pole. Finally, when $\left|Q_{2}\right|$ drops below some threshold, the vortex perturbation begins to decay algebraically (see curve 4 ) as for the $\delta=0$ case. Curve 4 with $r_{c}=6$ exhibits a small region of nonexponential decay at $t \approx 2500$ before decaying at the Landau pole value. The decay rates for $r_{c}=10$ and 9 (curves 2 and 3, respectively) both tend to the exponential decay rate given by the dominant Landau pole. At larger values of $r_{c}$, the amplitude threshold for the algebraic decay may be reached before this period of exponential decay occurs; in this case the exponential decay may not be observed.

The features in real space of a quasimode for a profile (18) with a defect are illustrated by Fig. 14, which can be compared with the purely Gaussian case in Fig. 1. This figure shows a $n=2$ disturbance to a profile with a defect at $r_{c}=2.85$. Again we see a combination of spiral wind-up coupled with the modal behavior, in which the elliptical distortion gives a sign of vorticity that depends on angle $\theta$ rather than on radius. At the early time, the picture in Fig. 14(a) is similar to the Gaussian case in Fig. 1(a) with the flat defect at $r_{c}=2.85$ clearly visible. However, at late times in Fig. 14(b) we note that the elliptical distortion is still present (unlike in the Gaussian case), showing that the quasimode 


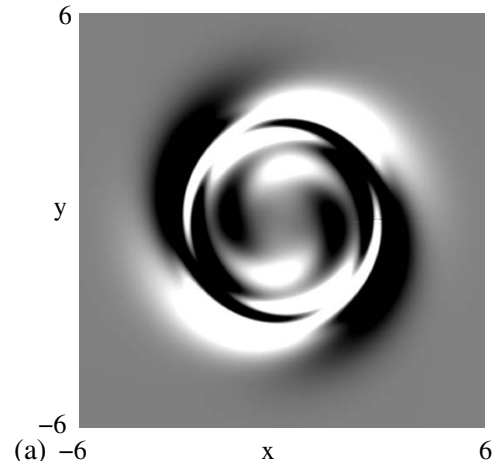

(a) -6

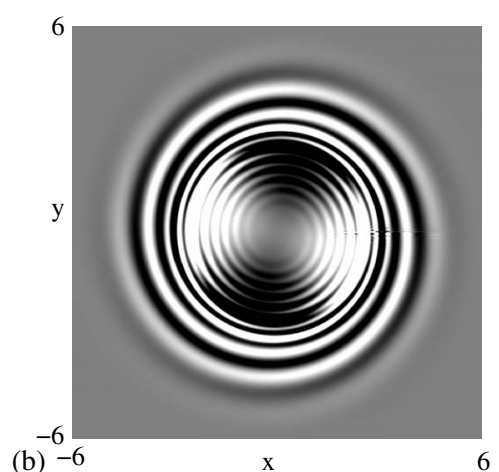

6
FIG. 14. An $n=2$ quasimode within a profile (18) with $\delta=1, \epsilon=0.3$, and $r_{c}=2.85$ at (a) $t=200$ and (b) $t=1000$. The gray scale is as in Fig. 1 . persists in this case, and we have a neutral mode. We can also see how the defect interacts with the vorticity in the neighborhood of $r_{c}=2.85$, where the spiral arms are broader. It is in this region where cat's eyes (of a width of order $\epsilon=0.3$ ) could be maintained in the full nonlinear problem.

\section{CONCLUSIONS}

In this paper we have studied how an axisymmetric vortex relaxes after being subjected to a weak, instantaneous, nonaxisymmetric forcing. This has been accomplished using a two-pronged numerical approach; one of these relies on the tracking of Landau poles in the complex plane while the other is a Keller-box strategy applied to the linear problem. Our underlying vortex has been taken to be a Gaussian type which has been modified by inclusion of zone where the profile is artificially flattened. After a transient, the nonaxisymmetric perturbation decays with a decay rate and frequency fixed by the Landau pole that has the least negative imaginary part. The time taken for the perturbation to start to decay at this Landau pole rate depends upon the structure of the axisymmetric profile.

For a profile with a flat region of thickness $\epsilon \lessgtr 0.35$ positioned at $r=r_{c}$, we have shown that two neutral modes exist at two distinct values of $r=r_{c}$. Moreover, in the limit as the defect thickness $\epsilon \rightarrow 0$, one of these neutral modes moves to the point $r_{c}=3.448$, in accord with the asymptotic results of Le Dizès. ${ }^{16}$ Thus, this study suggests that when cat's eyes below a critical thickness are generated in a vortex via a nonlinear mechanism, they can persist at two distinct radii. However, nonlinear mechanisms rarely give axisymmetric profiles as clean as the ones studied in this paper and the fine scale structure within the eyes often becomes significant. It is clear that further detailed numerical work is required to ascertain whether our conclusions can be extended to nonlinear situations.

\section{ACKNOWLEDGMENTS}

This work was undertaken on the EPSRC Grant No. EP/ D032202/1.
${ }^{1}$ B. Fornberg, "A numerical study of 2-D turbulence," J. Comput. Phys. 25, 1 (1977)

${ }^{2}$ J. C. McWilliams, "The emergence of isolated coherent vortices in turbulent flow," J. Fluid Mech. 146, 21 (1984).

${ }^{3}$ R. Benzi, G. Paladin, S. Patarnello, P. Santangelo, and A. Vulpiani, "Intermittency and coherent structures in two-dimensional turbulence," $\mathrm{J}$. Phys. A 19, 3771 (1986).

${ }^{4}$ M. E. Brachet, M. Meneguzzi, H. Politano, and P. L. Sulem, "The dynamics of freely decaying two-dimensional turbulence," J. Fluid Mech. 194, 333 (1988).

${ }^{5}$ T. A. Guinn and W. H. Schubert, "Hurricane spiral bands," J. Atmos. Sci. 50, 3380 (1993).

${ }^{6}$ G. B. Smith and M. T. Montgomery, "Vortex axisymmetrization: Dependence on azimuthal wave-number or asymmetric radial structure changes," Q. J. R. Meteorol. Soc. 121, 1615 (1995).

${ }^{7}$ K. Bajer and H. K. Moffatt, "On the effect of a central vortex on a stretched magnetic flux tube," J. Fluid Mech. 339, 121 (1997).

${ }^{8}$ M. V. Melander, J. C. McWilliams, and N. J. Zabusky, "Axisymmetrization and vorticity-gradient intensification of an isolated two-dimensional vortex through filamentation," J. Fluid Mech. 178, 137 (1987).

${ }^{9}$ R. J. Briggs, J. D. Daugherty, and R. H. Levy, "Role of Landau damping in crossed-field electron beams and inviscid shear flow," Phys. Fluids 13, 421 (1970).

${ }^{10}$ D. A. Schecter, D. H. E. Dubin, A. C. Cass, C. F. Driscoll, I. M. Lansky, and T. M. O'Neil, "Inviscid damping of asymmetries on a twodimensional vortex," Phys. Fluids 12, 2397 (2000).

${ }^{11}$ N. J. Balmforth, S. G. Llewellyn Smith, and W. R. Young, "Disturbing vortices," J. Fluid Mech. 426, 95 (2001).

${ }^{12}$ A. P. Bassom and A. D. Gilbert, "The spiral wind-up of vorticity in an inviscid planar vortex," J. Fluid Mech. 371, 109 (1998).

${ }^{13}$ N. R. Corngold, "Linear response of the two-dimensional pure electron plasma: Quasimodes for some model profiles," Phys. Plasmas 2, 620 (1995).

${ }^{14}$ C. Macaskill, A. P. Bassom, and A. D. Gilbert, "Nonlinear wind-up in a strained planar vortex," Eur. J. Mech. B/Fluids 21, 293 (2002).

${ }^{15} \mathrm{M}$. R. Turner and A. D. Gilbert, "Linear and nonlinear decay of cat's eyes in two-dimensional vortices, and the link to Landau poles," J. Fluid Mech. 593, 255 (2007).

${ }^{16}$ S. Le Dizès, "Non-axisymmetric vortices in two-dimensional flows," J. Fluid Mech. 406, 175 (2000).

${ }^{17}$ H. B. Keller, "A new difference scheme for parabolic problems," in $\mathrm{Nu}$ merical Solution of Partial Differential Equations, II (SYNSPADE 1970) (Proceedings of the Symposium), University of Maryland, College Park, MD, 1970 (Academic, New York, 1971).

${ }^{18}$ A. J. Bernoff and J. F. Lingevitch, "Rapid relaxation of an axisymmetric vortex," Phys. Fluids 6, 3717 (1994).

${ }^{19}$ J. F. Lingevitch and A. J. Bernoff, "Distortion and evolution of a localized vortex in an irrotational flow," Phys. Fluids 7, 1015 (1995).

${ }^{20}$ P. J. Schmid and D. S. Henningson, Stability and Transition in Shear Flows (Springer, New York, 2001). 
Physics of Fluids is copyrighted by the American Institute of Physics (AIP). Redistribution of journal material is subject to the AIP online journal license and/or AIP copyright. For more information, see http://ojps.aip.org/phf/phfor.jsp 